\title{
Proposta de atividade didática teórica e experimental de telescópios refratores e suas aplicações
}

Proposal of a theoretical and experimental didactical practice of refracting telescopes and their applications

\author{
L. F. G. Dib ${ }^{1}$, E. A. Barbosa*1®, F. T. Degasperi ${ }^{1}$ \\ ${ }^{1}$ Centro Estadual de Educação Tecnológica Paula Souza (CEETEPS) Unidade de Pós-Graduação, Extensão e Pesquisa, São \\ Paulo, SP, Brasil.
}

Recebido em 05 de março de 2020. Revisado em 29 de abril de 2020. Aceito em 07 de maio de 2020.

\begin{abstract}
Uma atividade didática para o estudo teórico e experimental do princípio de funcionamento do telescópio refrator é proposta para cursos de graduação em física, tecnologia e engenharia. Utilizou-se o formalismo matricial para determinar as propriedades do telescópio, como a distância entre as lentes e a magnificação, em função dos comprimentos focais das lentes objetiva e ocular e da distância objeto-telescópio. Na parte experimental, foi montado um arranjo com o telescópio em si e um olho artificial, composto de uma lente positiva como cristalino e um anteparo plano como retina. A magnificação foi medida diretamente da retina do olho artificial e comparada, à luz dos cálculos de propagação de incertezas, com medidas obtidas com o auxílio de expressões extraídas do método matricial.
\end{abstract}

Palavras-chave: telescópio, óptica matricial, magnificação, lentes delgadas.

A didactical activity aiming the theoretical and experimental study of the refractive telescope is proposed for undergraduate physics, technology and engineering courses. The matrix method was used for determining the telescope properties like distance between the lenses and magnification, as a function of the lenses focal lengths and the distance between the object and the telescope. In the experiments the optical setup was comprised by the telescope itself and an artificial eye, which in turn is comprised by a positive lens as the eye lens and a planar screen as the retina. The magnification was directly measured from the retina and compared with the measurements obtained with the help of the equations retrieved from the matrix method, concerning the uncertainty propagation. Keywords: telescope, matrix optics, magnification, thin lenses.

\section{Introdução}

Desde o século XVI, o uso do telescópio é de inquestionável importância para a observação de fenômenos astronômicos e também possibilitou importantes descobertas e observações de fenômenos terrestres. Após séculos de aperfeiçoamento, os telescópios evoluíram muito e hoje eles vão muito além de apenas conjuntos de lentes. Possuem grande tecnologia embarcada e são utilizados das formas mais variadas. Diversos arranjos são utilizados, não se limitando mais à observação apenas de luz visível. Atualmente é possível, por exemplo, fazer observações de comprimentos de onda de infravermelho e raios gama [1]. Os telescópios estão presentes tanto em observatórios, como em satélites e sondas espaciais. Todas essas tecnologias se derivaram dos telescópios de Kepler e de Galileu, ambos basicamente concebidos com duas lentes, a lente objetiva e a lente ocular, sendo a primeira de menor potência que a segunda. O telescópio kepleriano tem as duas lentes positivas, enquanto que no galileano a lente objetiva é positiva e a ocular é negativa. Este segundo arranjo passou a ser muito utilizado para observar obje-

*Endereço de correspondência: ebarbosa@fatecsp.br tos na Terra, com a construção de lunetas e binóculos. Devido à utilização da lente divergente, o comprimento do tubo fica mais compacto e a imagem formada não é invertida. Ideal para transportar com mais facilidade e para observar objetos em terra na posição correta.

O uso de telescópios é importante não somente para fins científicos, mas também em uma série de aplicações voltadas à engenharia. Instrumentos de medição voltados à agrimensura e topografia, como teodolitos e taqueômetros, são baseados em telescópios; outras variantes deste instrumento, como binóculos, periscópios e rangefinders são largamente utilizados em atividades de engenharia militar.

Arranjos ópticos frequentemente são compostos por uma série de elementos como lentes, espelhos e prismas, dispostos ao longo de um eixo óptico. A descrição da trajetória do raio de luz pelas diversas lentes, espaços livres e interfaces envolve cálculos sucessivos e repetitivos. $\mathrm{O}$ uso de matrizes pode diminuir significativamente o trabalho de se calcular características de imagens feitas por sistemas ópticos complexos, sempre no regime paraxial, no qual os raios luminosos fazem pequenos ângulos com o eixo óptico e as equações de traçado de raios são lineares 
nas coordenadas de posição e de ângulo. Este formalismo aplica-se muito frequentemente a cálculos preliminares de sistemas ópticos complexos [2 5$]$ e ao projeto e dimensionamento de ressonadores laser 6 8], incluindo o cálculo de cavidades laser para o regime de Kerr-lens modelocking (KLM) 911, entre outras aplicações.

Neste artigo, demonstramos o cálculo de parâmetros do telescópio, como magnificação angular e distância entre lentes, através do método matricial, também chamado de método ABCD. A magnificação angular do telescópio foi determinada como sendo igual a um dos elementos de matriz (d) do sistema. Descrevemos uma atividade experimental didática para a verificação dos resultados teóricos por meio da montagem, em bancada óptica, de um sistema composto de um telescópio refrator - composto apenas por lentes, em contrapartida ao telescópio refletor, constituído de pelo menos um espelho esférico e um olho observador. O telescópio é formado por uma lente objetiva, uma lente ocular e um espaço livre entre ambas. O olho é composto de um anteparo plano e opaco, que faz as vezes da retina, e de uma lente positiva, que atua como o cristalino. $\mathrm{Na}$ atividade experimental ora proposta realizamos uma medida impossível em situações reais, a medida do tamanho das imagens formadas na retina do olho observador nu (sem o telescópio) e com o telescópio. Comparamos os resultados desta medida direta com os resultados obtidos com o auxílio do método matricial e discutimos as condições dentro das quais estes últimos são válidos.

\section{Método Matricial (ABCD) de Cálculo de Sistemas Ópticos}

O uso de matrizes pode diminuir significativamente o trabalho de se calcular características de imagens feitas por sistemas ópticos complexos no regime paraxial. Um sistema óptico é usualmente formado por lentes, prismas, espelhos e outros componentes e destina-se a formar imagens ou fazer parte de um sistema de iluminação. A Figura 1 representa um sistema que pode combinar todos estes componentes. Um raio luminoso, ao passar pelo sistema, tem como parâmetros mais importantes a sua coordenada vertical $y$ e a sua coordenada angular $\theta$. De posse destes parâmetros, pode-se determinar o caminho dos raios ao longo de um sistema óptico e obter as características de uma imagem, por exemplo. De forma recorrente, pode-se descrever a passagem do raio por um determinado sistema óptico dentro do regime paraxial no qual $\operatorname{sen} \boldsymbol{\theta} \approx \boldsymbol{t g} \boldsymbol{\theta} \boldsymbol{\theta}-$ pelas equações

$$
\begin{aligned}
& y^{\prime}=A y+B \theta \\
& \theta^{\prime}=C y+D \theta
\end{aligned}
$$

onde $y$ e $\theta$ são as coordenadas do raio à entrada do sistema óptico, enquanto que $y^{\prime}$ e $\theta$ ' são as coordenadas à saída do sistema. A equação (1) pode ser expressa na

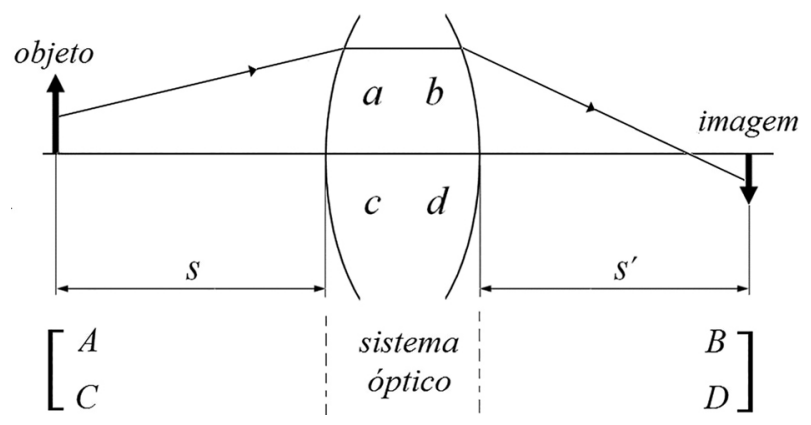

Figura 1: Raios emergem do objeto, passam pelo sistema óptico caracterizado pelos elementos de matriz $a, b, c$ e $d$ e formam a imagem

forma matricial

$$
\left[\begin{array}{l}
y^{\prime} \\
\theta^{\prime}
\end{array}\right]=\left[\begin{array}{ll}
A & B \\
C & D
\end{array}\right]\left[\begin{array}{l}
y \\
\theta
\end{array}\right]
$$

Os elementos de matriz A, B, C e D estão relacionados a características do sistema óptico, como extensão, vergência e propriedades de simetria.

Na Figura 1, um objeto encontra-se a uma distância $s$ de um sistema óptico genérico caracterizado por quatro elementos de matriz $a, b, c e d$. Nosso interesse inicial é obter uma expressão que relacione a distância $s$ ' entre o sistema óptico e a imagem com o valor de se os parâmetros do conjunto óptico. Vale ressaltar a distinção entre os elementos de matriz $a, b, c$ e $d$, referentes exclusivamente ao sistema óptico, e os elementos $A, B, C$ e $D$, relacionados à trajetória dos raios do objeto até a imagem.

Sabemos que a relação entre um ponto no plano objeto e sua respectiva imagem é unívoca, ou seja, a cada ponto no plano objeto corresponde um único ponto no plano imagem. De acordo com a teoria de óptica matricial, a matriz geral $A B C D$ é obtida, no caso mostrado na Figura 1, pelo produto de três matrizes: a matriz de caminho livre $S^{\prime}$ devido à propagação do raio pela distância $s$ ' , a matriz $\mathbb{M}$ devido à passagem da luz pelo sistema óptico em si, e a matriz de caminho livre $S$, que descreve a propagação da luz entre o objeto e a face esquerda do sistema óptico. Sendo $S=\left[\begin{array}{ll}1 & s \\ 0 & 1\end{array}\right], S^{\prime}=\left[\begin{array}{ll}1 & s^{\prime} \\ 0 & 1\end{array}\right] \mathrm{e}$ $\mathbb{M}=\left[\begin{array}{ll}a & b \\ c & d\end{array}\right] 12$, tem-se

$$
\begin{aligned}
& {\left[\begin{array}{ll}
A & B \\
C & D
\end{array}\right]=S^{\prime} \times \mathbb{M} \times S} \\
& =\left[\begin{array}{ll}
1 & s^{\prime} \\
0 & 1
\end{array}\right] \times\left[\begin{array}{ll}
a & b \\
c & d
\end{array}\right] \times\left[\begin{array}{ll}
1 & s \\
0 & 1
\end{array}\right]
\end{aligned}
$$

de onde se obtêm as expressões

$$
\begin{gathered}
A=a+s^{\prime} c \\
B=a s^{\prime} s+s^{\prime} b+c s+d
\end{gathered}
$$




$$
\begin{gathered}
C=c \\
D=c s+d
\end{gathered}
$$

Consideremos na Figura 2 um raio originado do ponto $P(\operatorname{com} \theta \neq 0)$, de alturay $=0$ no plano objeto, que atinge o ponto $P^{\prime}$ de altura $y^{\prime}=0$ no plano imagem. A conveniência de se escolher o raio de trajetória $P P^{\prime}$ vem do fato de que tanto $y$ quanto $y$ ' são nulos, o que facilita significativamente a resolução do problema através da equação 1a:

$$
y^{\prime}=A y+B \theta \Longrightarrow 0=A .0+B \theta \Longrightarrow B=0
$$

Da condição acima, obtém-se, da equação (3b), a relação

$$
s^{\prime}=-\frac{a s+b}{c s+d}
$$

A equação (4) relaciona as distâncias objeto-sistema e imagem-sistema com os seus elementos de matriz, sendo aplicável, em princípio, a qualquer sistema óptico.

\subsection{Telescópio kepleriano}

Como já indica o prefixo grego "tele", este instrumento se aplica a observar imagens de objetos distantes. $\mathrm{O}$ telescópio é formado basicamente por uma lente objetiva e uma lente ocular. As lentes são posicionadas de maneira que a imagem real formada pela objetiva situa-se no plano focal dianteiro da ocular, como mostra o esquema básico do conjunto mostrado na Figura 3a. Por este motivo, os raios oriundos de cada ponto desta imagem real emergem da ocular propagando-se paralelamente entre si. Quando o olho é posicionado logo atrás da ocular, o cristalino converge estes raios paralelos na retina. A Figura $3 \mathrm{~b}$ mostra o que ocorre quando um observador aproxima

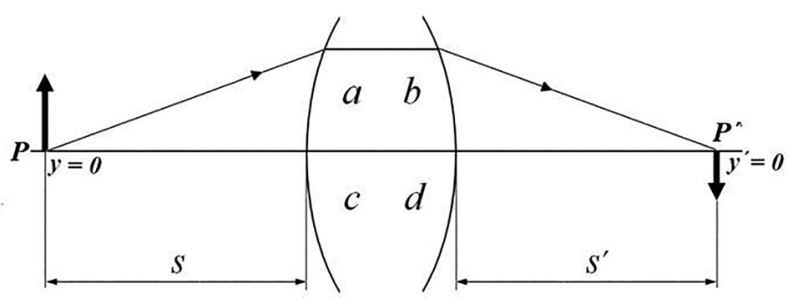

Figura 2: Caminho do raio originado em $y=0$ (ponto $\mathrm{P}$ ), atingindo o plano imagem em $y^{\prime}=0$ (ponto $\mathrm{P}^{\prime}$ ) seu olho da lente ocular: admitindo-se, como hipótese simplificadora, que a distância entre o cristalino e a ocular é nula, a imagem formada na retina do observador é direta, o que leva à conclusão de que o observador que observa o objeto através do telescópio vê a sua imagem de cabeça para baixo.

O que se convencionou popularmente chamar de aumento do telescópio é definido pela razão entre o tamanho $h$ ' ' da imagem formada na retina pelo olho através do instrumento óptico e o tamanho $h$ ' da imagem formada na retina pelo olho nu, também chamada de magnificação:

$$
M_{r e t}=\frac{h^{\prime \prime}}{h^{\prime}}
$$

Uma relação equivalente à da equação (5) é a razão entre o ângulo de observação $\alpha^{\prime}$ de um objeto através do sistema óptico e o ângulo de observação $\alpha$ do mesmo objeto a olho nu [13 14], chamada de magnificação angular:

$$
M=\frac{\alpha^{\prime}}{\alpha}
$$

A magnificação angular do telescópio pode ser facilmente obtida pelo método matricial. Para usarmos a equação (6), consideremos antes a situação típica em que a distância entre o objeto observado e o telescópio seja muito maior que a abertura da objetiva. Por exemplo, em telescópios usados em astronomia, a distância entre o astro observado e o telescópio é infinitamente maior que o diâmetro dos espelhos dos maiores telescópios em construção hoje em dia (da ordem de dezenas de metros). Assim, é plenamente razoável considerar a aproximação na qual todos os raios oriundos do objeto que atingem a lente são paralelos. Por este mesmo motivo, é igualmente aceitável considerar que os raios provenientes de um ponto do objeto atingem o telescópio e o olho nu sob o mesmo ângulo $\alpha$, como mostrado na Figura 4: a distância $x$ entre o objeto e a lente objetiva é muito maior que a distância $L$ entre as lentes objetiva e ocular, de modo que, se o objeto tem altura $h$, o ângulo de entrada no olho em radianos é $\alpha=h / x$ (Figura 4a) muito próximo do ângulo $h /(x-L)$ (Figura 4b) de entrada no telescópio.

A matriz que descreve a passagem de raios paraxiais por uma lente delgada de comprimento focal $f$ é $\left[\begin{array}{cc}1 & 0 \\ -1 / f & 1\end{array}\right][12$. Desta forma, a matriz do telescópio composto de uma lente objetiva de comprimento focal
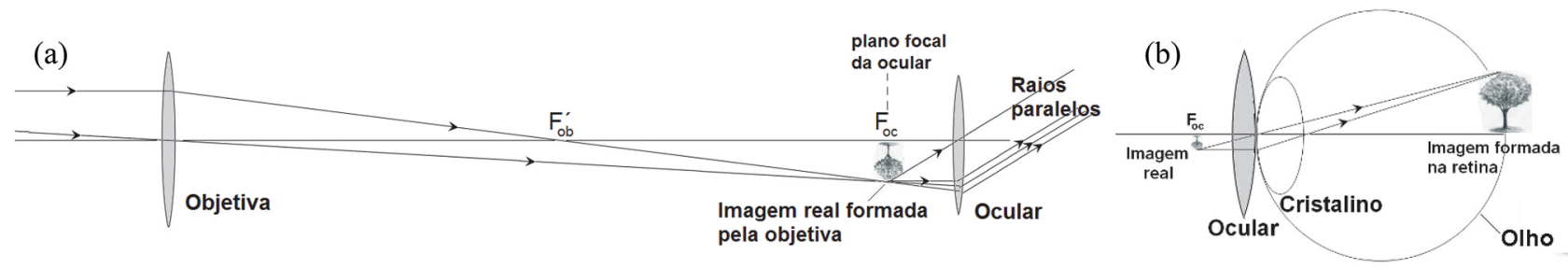

Figura 3: a - Caminho dos raios propagando-se propagando-se pelas lentes objetiva e ocular; b- caminho dos raios entre a imagen real antes da ocular e a imagem projetada na retina 
$f_{o b}$, uma lente ocular de comprimento focal $f_{o c}$, ambas consideradas delgadas, e um espaço livre $L$ entre ambas terá os elementos abaixo:

$\left[\begin{array}{ll}a & b \\ c & d\end{array}\right]=\left[\begin{array}{cc}1 & 0 \\ -1 / f_{o c} & 1\end{array}\right] \times\left[\begin{array}{ll}1 & L \\ 0 & 1\end{array}\right] \times\left[\begin{array}{cc}1 & 0 \\ -1 / f_{o b} & 1\end{array}\right]$

de onde se obtêm os elementos de matriz

$$
\begin{gathered}
a=1-L / f_{o b} \\
b=L \\
c=-1 / f_{o b}-1 / f_{o c}+L /\left(f_{o b} f_{o c}\right) \\
d=1-L / f_{o c}
\end{gathered}
$$

Reescrevemos a equação (1) para relacionar os valores de altura e ângulo dos raios que emergem e que incidem no telescópio:

$$
\begin{aligned}
& y^{\prime}=a y+b \alpha=\left(1-L / f_{o b}\right) y+L \alpha \\
\alpha^{\prime}= & c y+d \alpha=\left[-1 / f_{o b}-1 / f_{o c}+L /\left(f_{o b} f_{o c}\right)\right] y \\
+ & \left(1-L / f_{o c}\right) \alpha
\end{aligned}
$$

A magnificação angular é dada pela razão $\alpha^{\prime}{ }_{t e l} / \alpha$, de modo que o raio mais conveniente para determiná-la através da equação $8 \mathrm{~b}$ é aquele para o qual $y=0$, mostrado na Figura 5. Desta maneira, a relação $\alpha^{\prime}=$ $c y+d \alpha$ toma a forma

$$
\alpha^{\prime}{ }_{t e l}=d \alpha \Rightarrow d=\frac{\alpha^{\prime}{ }_{t e l}}{\alpha}
$$

Comparando-se as equações (6d) e (9), conclui-se que a magnificação angular do telescópio é igual ao elemento $d$ da sua matriz:

$$
M_{t e l}=\frac{\alpha^{\prime}{ }_{t e l}}{\alpha}=d=1-\frac{L}{f_{o c}}
$$

A distância $L$ entre os dois espelhos se determina considerandose que os raios originados de um ponto no plano objeto e que emergem da lente ocular são paralelos; isto equivale a dizer que a distância $s$ ' tende a infinito. Assim, da , equação (4) obtém-se a condição

$$
c s+d=0
$$

Das equações (7c), (7d) e (11), obtém-se a distância $L$ entre as lentes objetiva e ocular do telescópio como

$$
L=f_{o c}+\frac{s f_{o b}}{s-f_{o b}},
$$

De forma que a magnificação é obtida da equação 10 como

$$
M_{t e l}=-\frac{s}{s-f_{o b}} \frac{f_{o b}}{f_{o c}}
$$

Arranjo confocal - um arranjo muito comum, principalmente em telescópios astronômicos, é o confocal, no qual sobrepõem-se o ponto focal traseiro da objetiva e o ponto focal dianteiro da ocular, como mostrado na Figura 6 . Esta configuração é usada quando o objeto observado encontra-se no infinito. Na Figura 6a, os raios incidentes propagam-se paralelamente ao eixo óptico e convergem no ponto focal da objetiva, divergindo a partir do ponto focal dianteiro da ocular. $\mathrm{Na} 6 \mathrm{~b}$, o feixe colimado incidente propaga-se obliquamente em relação ao eixo óptico, converge em um ponto no plano focal traseiro da objetiva e emerge da lente ocular também colimado, já que ele diverge a partir do plano focal dianteiro da ocular. Já que a configuração da Figura 6 vale para $s \rightarrow \infty$, a equação (12) toma a forma

$$
L=\lim _{s \rightarrow \infty}\left(f_{o c}+\frac{s f_{o b}}{s-f_{o b}}\right)=f_{o b}+f_{o c}
$$

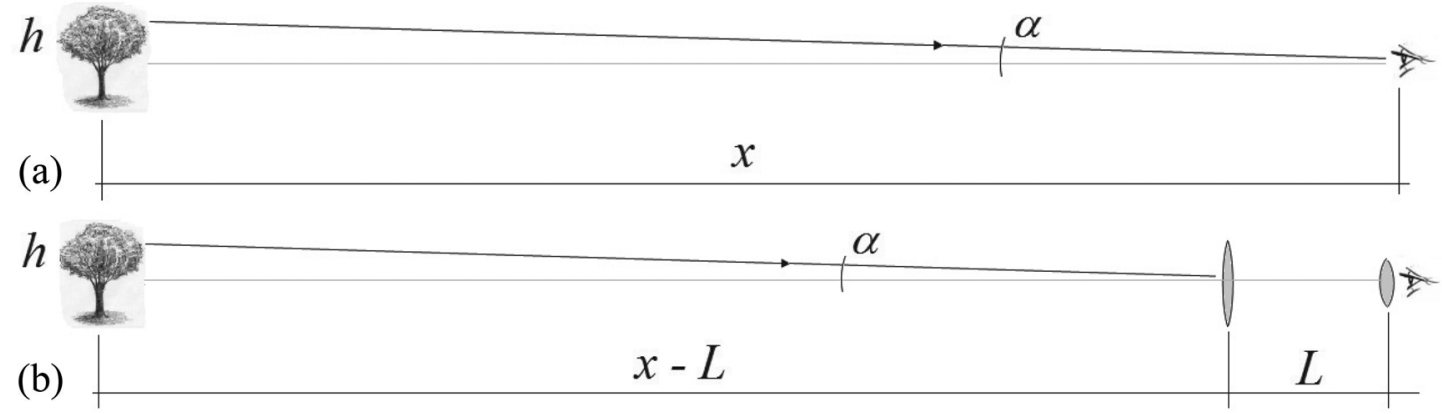

Figura 4: a - ângulo de entrada no olho nu e b - ângulo de entrada no olho por meio do telescópio

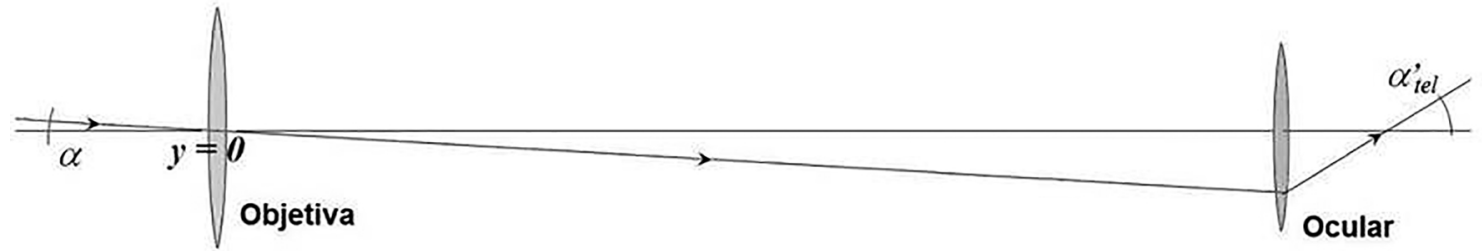

Figura 5: Percurso do raio que atinge o telescópio em $y=0$ 


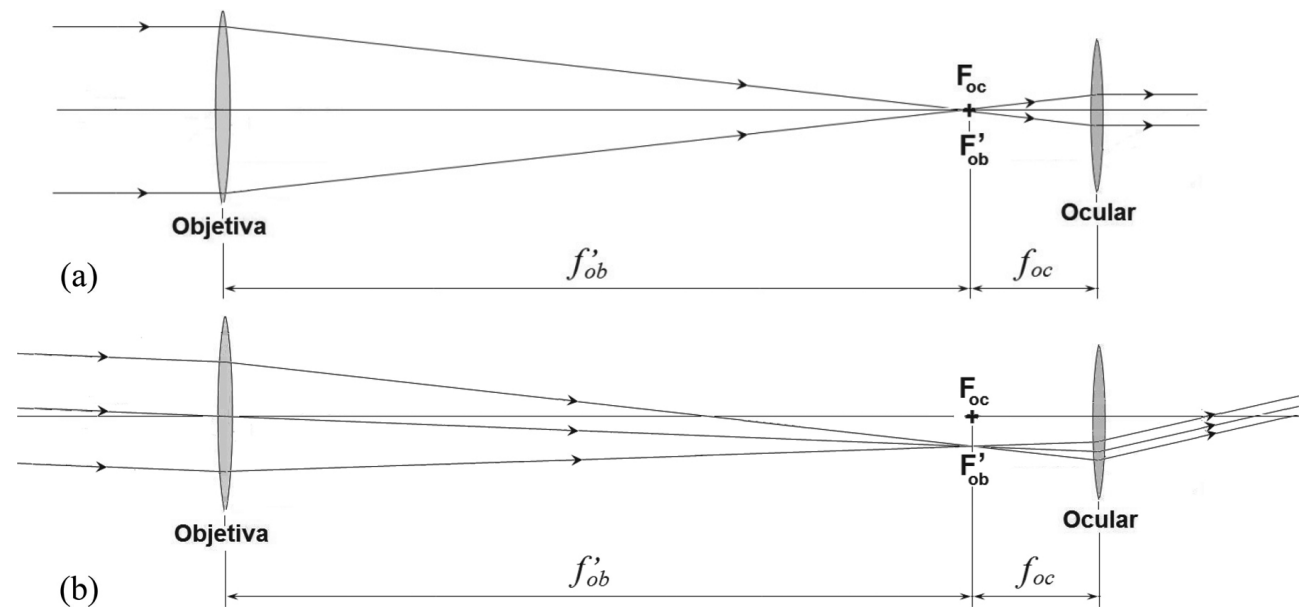

Figura 6: Propagação de raios no telescópio confocal; a - raios paralelos ao eixo óptico; b - raios oblíquos em relação ao eixo óptico

Procede-se analogamente para se determinar a magnificação na configuração confocal:

$$
M_{t e l}=\lim _{s \rightarrow \infty}\left(-\frac{s}{s-f_{o b}} \frac{f_{o b}}{f_{o c}}\right)=-\frac{f_{o b}}{f_{o c}}
$$

A equação (14) expressa o já esperado resultado para $L$ como a soma dos comprimentos focais da objetiva e ocular na configuração confocal, enquanto que a equação (15) indica que um telescópio, para ter alta magnificação angular, deve ter uma lente objetiva com o maior comprimento focal possível, e uma lente ocular com o menor comprimento focal possível, condições válidas para qualquer arranjo de telescópio. Além disso, sendo $f_{o b} f_{o c}>0$, a magnificação é negativa, o que corrobora o indicado nas Figuras $3 \mathrm{~b}$ e $6 \mathrm{~b}$, de que a imagem vista pelo telescópio de Kepler é invertida. Se o telescópio é terrestre, há a necessidade de inserção de algum conjunto eretor entre as lentes objetiva e ocular, como, por exemplo, um par de lentes positivas de mesma potência, ou um prisma de Porro 13.

\subsection{Telescópio galileano}

Este telescópio emprega uma lente objetiva positiva e uma lente ocular negativa. As equações (7-15) também são válidas para este telescópio, desde que $f_{o c}<0$. Das equações 14 e 15, conclui-se que o telescópio de Galileu é mais compacto que o de Kepler, já que a distância entre as lentes do primeiro é $L_{\infty}=f_{o b}-\left|f_{o c}\right|$, e que a sua magnificação é positiva, proporcionando ao observador uma imagem direta, sem a necessidade de sistemas eretores. As Figuras 7a e 7b mostram, analogamente à Figura 6, o traçado de raios no telescópio de Galileu em arranjos
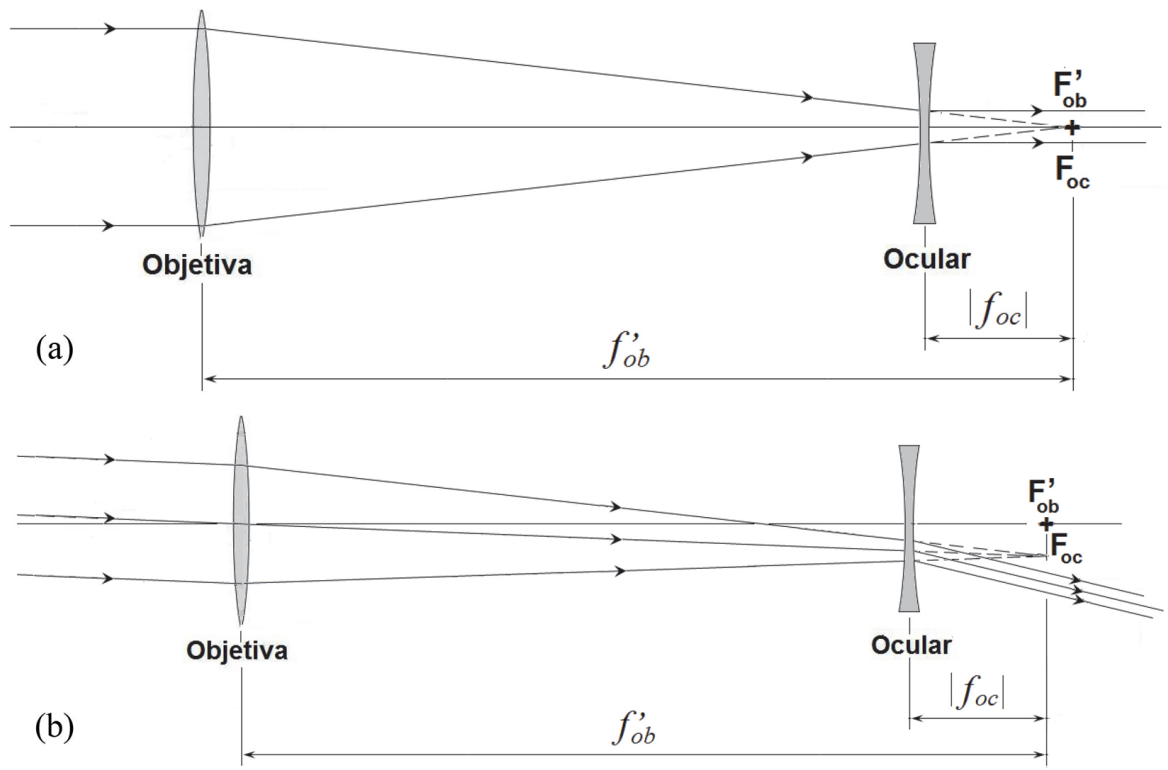

Figura 7: Propagação dos raios no telescópio galileano; a - raios paralelos ao eixo óptico; b - raios oblíquos em relação ao eixo óptico 
confocais. Nesta Figura, o módulo do comprimento focal da lente ocular é o mesmo do da ocular na Figura 6.

\section{Procedimento experimental}

Foi construído um modelo de objeto com 3 LEDs brancos, alimentados com uma fonte de $3,0 \mathrm{~V}$, que são posicionados em uma régua de acrílico, de forma não simétrica, a fim de permitir identificar possíveis inversões de imagem, como mostra a Figura 8. A distância entre os LEDs mais próximos é $9 \mathrm{~cm}$, e a distância entre o LED do meio e o mais distante é $17 \mathrm{~cm}$.

A bancada óptica deve ser posicionada à maior distância possível do objeto para garantir o regime paraxial, situação típica envolvendo telescópios. No caso, a distância objeto-objetiva é $s \sim 880 \mathrm{~cm}$. O modelo do olho do observador foi montado utilizando-se uma lente convergente de distância focal $15,4 \mathrm{~cm}$ e um anteparo opaco no seu plano imagem, ambos posicionados em um único suporte. A lente desempenha o papel de cristalino e o anteparo faz as vezes da retina, quando o objeto está $\sim 880 \mathrm{~cm}$ do cristalino, o que resulta numa distância cristalino-retina de $15,8 \mathrm{~cm}$, mantida fixa em todas as etapas do experimento. Para esta configuração, mostrada na Figura 9, admite-se que o olho do observador está relaxado. Nesta etapa, mede-se o tamanho $h$ ' da imagem formada na retina pelo olho nu. Essa informação será utilizada para calcular a magnificação do telescópio.

\subsection{Telescópio kepleriano}

Para este telescópio, usou-se uma lente ocular de comprimento focal 9,4 cm e uma lente objetiva de comprimento focal $66,7 \mathrm{~cm}$. A ocular foi posicionada o mais próximo

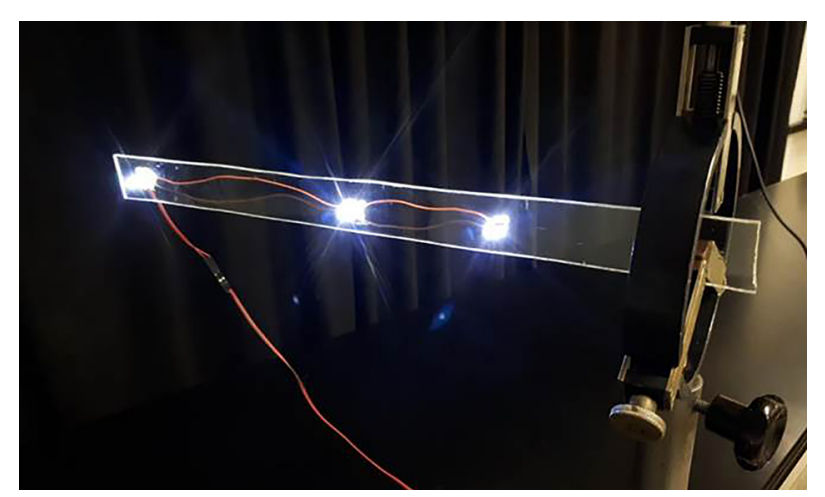

Figura 8: Objeto formado por arranjo de LEDs

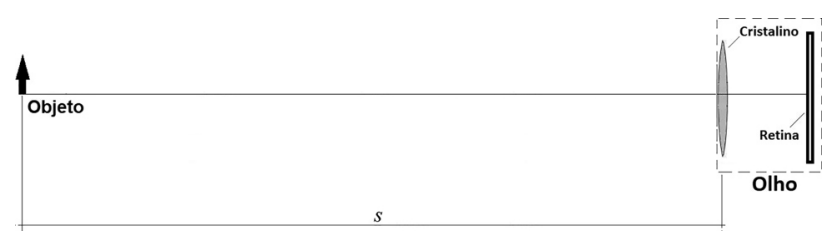

Figura 9: Esquema do "olho" relaxado, formado pelo "cristalino" (lente positiva) e pela "retina" (anteparo opaco) possível ao modelo do olho para emular a configuração mostrada na Figura 3b. A Figura 10 mostra o arranjo.

A lente ocular deve ser posicionada a uma distância $f_{\text {oc }}$ da imagem real formada pela lente objetiva, e o olho deve ser colocado à menor distância possível da ocular. Pelo fato de as lentes não serem perfeitamente delgadas e por eventuais dificuldades de se obter distância nula entre a ocular e o cristalino, a imagem na retina pode sair desfocada. Para solucionar este problema, translada-se de forma solidária o conjunto ocular + olho até focalizarse a imagem na retina. O tamanho da imagem $h$ ' ' na retina com o olho vestido deve ser medido para calcular a magnificação do telescópio.

O comprimento $L$ entre as lentes do telescópio também é medido nesta etapa.

\subsection{Telescópio galileano}

Para esse telescópio, trocou-se a lente ocular convergente por uma lente divergente com distância focal $f_{o c}=$ -9,5 cm, de módulo o mais próximo possível ao da ocular do modelo anterior, para pemitir melhor comparação de resultados. Assim como no caso anterior, ela foi posicionada o mais próximo possível do olho. $\mathrm{O}$ alinhamento do conjunto foi feito de forma similar ao caso do telescópio kepleriano, de forma que a distância objeto-objetiva foi de $s-L=816,2 \mathrm{~cm}$. O arranjo está mostrado na Figura 11.

Uma vez alinhado o sistema, mediu-se o tamanho $h$ ' ' da imagem formada na retina e a distância $L$ entre a lente objetiva e a lente ocular.

\section{Resultados e discussão}

No intuito de se manter o experimento o mais simples possível, as distâncias entre as imagens dos LEDs formadas na retina pelo olho nu e pelo olho vestido foram medidos com um paquímetro de precisão $0,05 \mathrm{~mm}$. Por não se tratar de um processo de medição convencional, no

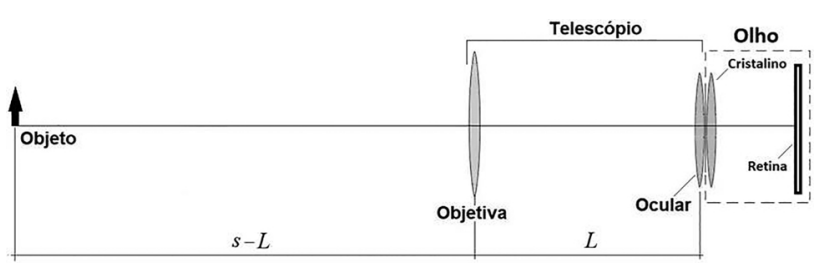

Figura 10: Arranjo do telescópio kepleriano com o olho

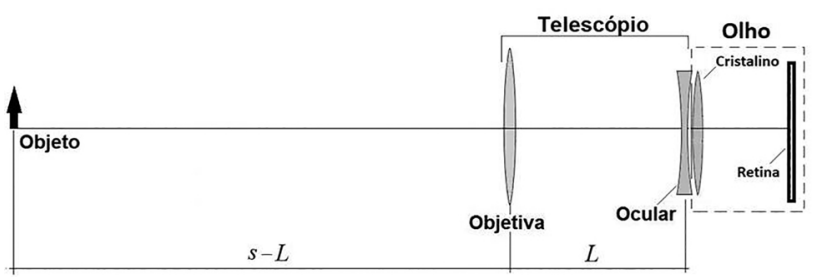

Figura 11: Arranjo do telescópio galileano com o olho 
qual há contato entre o objeto e os bicos de medição do paquímetro, adotou-se uma postura conservadora para estabelecer a incerteza da medida, adotada como 0,2 $\mathrm{mm}$. Esta postura foi mantida nas medições dos demais comprimentos: os valores de $L$ foram medidos com uma trena de precisão $1 \mathrm{~mm}$, e a incerteza foi adotada como $2 \mathrm{~mm}$, por levar em conta a dificuldade de se medir a distância entre duas lentes sobre a bancada óptica; devido a eventuais deformações da trena na medição da distância objeto-objetiva, atribuiu-se à distância $s-L$ a incerteza de $5 \mathrm{~mm}$. Os resultados estão mostrados nas tabelas 1 e 2 . O erro percentual foi definido como $\varepsilon(\%)=\left|M_{r e t}-M_{t e l}\right| \times 100 / M_{t e l}$. Tamanhos de imagens formadas acima do eixo óptico têm sinais positivos, e vice-versa.

Tanto para o telescópio kepleriano quanto para o galileano, nota-se ótima concordância dos resultados de magnificação medida diretamente na retina $\left(M_{r e t}\right.$, eq. 5) com os resultados obtidos por meio das equações (10) e (13). Observando-se os resultados e suas respectivas incertezas nota-se a intersecção dos intervalos que contém os valores obtidos por estas três equações. Esta concordância mostra que a aproximação paraxial que permitiu a utilização do método ABCD foi adequada para o cálculo das características do sistema óptico, corroborado pelo fato de que o maior ângulo entre os raios e o eixo óptico foi $\sim 5^{\circ}$.

As imagens dos LEDs mais próximos foram usadas para determinar a magnificação $M_{r e t}$, por se adequarem melhor ao regime paraxial, e, justamente por este motivo, fornecerem imagens de melhor definição e qualidade. Os LEDs das extremidades, por serem mais distantes entre si, geraram imagens com coma [11] acentuada, não tendo sido, desta forma, utilizados para a medição dos tamanhos das imagens e suas respectivas magnificações. Sua utilização ficou restrita a demonstrar a inversão da imagem pelo telescópio kepleriano.

Este experimento permite a discussão e a abordagem de alguns importantes aspectos conceituais e instrumen-

Tabela 1: Resultados com o telescópio kepleriano

\begin{tabular}{lccl}
\cline { 1 - 1 } \multicolumn{3}{c}{ Telescópio kepleriano } & \\
\cline { 1 - 3 }$h^{\prime}(\mathrm{cm})$ & $h^{\prime \prime}(\mathrm{cm})$ & $M_{\text {ret }}$ (eq. 5) & \\
$-0,34 \pm 0,01$ & $2,52 \pm 0,01$ & $-7,4 \pm 0,2$ & \\
$L(\mathrm{~cm})$ & $f_{o c}(\mathrm{~cm})$ & $M_{\text {tel }}$ (eq. 10) & \\
$81,2 \pm 0,2$ & $9,5 \pm 0,2$ & $-7,55 \pm 0,15$ & $\varepsilon(\%)=2,0$ \\
$s-L(\mathrm{~cm})$ & $f_{o b}(\mathrm{~cm})$ & $M_{\text {tel }}($ eq. 13) & \\
$796,0 \pm 0,5$ & $67,0 \pm 0,5$ & $-7,7 \pm 0,2$ & $\varepsilon(\%)=4,0$ \\
\hline
\end{tabular}

Tabela 2: Resultados com o telescópio galileano

\begin{tabular}{lccl}
\cline { 1 - 1 } \multicolumn{3}{c}{ Telescópio galileano } & \\
\cline { 1 - 3 }$h^{\prime}(\mathrm{cm})$ & $h^{\prime \prime}(\mathrm{cm})$ & $M_{\text {ret }}$ (eq. 5) & \\
$-0,34 \pm 0,01$ & $-2,55 \pm 0,01$ & $7,5 \pm 0,2$ & \\
$L(\mathrm{~cm})$ & $f_{o c}(\mathrm{~cm})$ & $M_{\text {tel }}($ eq. 10) & \\
$61,0 \pm 0,2$ & $-9,4 \pm 0,2$ & $7,5 \pm 0,2$ & $\varepsilon(\%)=0,0$ \\
$s-L(\mathrm{~cm})$ & $f_{\text {ob }}(\mathrm{cm})$ & $M_{\text {tel }}$ (eq. 13) & \\
$816,2 \pm 0,5$ & $67,0 \pm 0,5$ & $-7,7 \pm 0,2$ & $\varepsilon(\%)=2,7$ \\
\hline
\end{tabular}

tais. O manuseio das lentes, sua montagem e ajuste na bancada óptica propicia ao estudante uma melhor compreensão do processo de formação de imagens e do projeto do telescópio, além de facilitar análises quantitativas, algo que a simples observação de imagens por um telescópio comercial, já montado, e com lentes encerradas em um tubo, não proporciona. A montagem na bancada permite a visualização da imagem real intermediária, formada pela lente objetiva no plano focal da ocular, e permite ao estudante entender o processo de obtenção e observação das imagens ao se distanciar ou aproximar as lentes do instrumento; a observação desta imagem real e a comparação de seu tamanho com a abertura relativa da lente ocular (razão entre o diâmetro e o comprimento focal da lente) facilita o entendimento do surgimento de coma quando a imagem dos dois LEDs extremos é formada. A montagem do telescópio em bancada óptica também apresenta a óbvia vantagem de permitir a montagem de vários kits experimentais e menos alunos por kit, algo que seria muito oneroso se fossem utilizados telescópios comerciais.

Durante a atividade, uma vez encerrada a etapa de medidas, os estudantes podem ser orientados a remover o olho artificial e observar pelo telescópio por seus próprios olhos, constatando que o ajuste do telescópio permite a observação com qualquer olho, desde que livre de anomalias como miopia e hipermetropia e ajustado para observar objetos no infinito.

\section{Conclusões}

Uma proposta de prática didática teórica e experimental de telescópio refrator foi apresentada, visando sua aplicação em disciplinas de física ou óptica para graduação em física, tecnologia e engenharia. Utilizou-se o formalismo matricial para determinar expressões que fornecem parâmetros característicos dos telescópios kepleriano e galileano, como a distância entre as lentes e a magnificação angular. Na parte experimental, cada telescópio foi montado junto a um olho artificial, o que permitiu a medição da magnificação diretamente na retina e a comparação deste resultado com outros resultados de magnificação, obtidos através do método matricial. Mostrou-se que esta atividade didática permite demonstrar uma aplicação prática e concreta do método matricial, além de propiciar a discussão sobre as condições nas quais este método é válido, à luz da análise da propagação e incertezas.

\section{Referências}

[1] M.W. Werner, T.L. Roellig, F.J. Low, G.H. Rieke, M. Rieke, W.F. Hoffmann, E. Young, J.R. Houck, B. Brand e G.G. Fazio, The Astrophysical Journal Supplement Series 154, 1 (2003).

[2] D.C. O'Shea, Elements of Modern Optical Design (Wiley, New York, 1985), v. 2. 
[3] B.E.A. Saleh e M.C. Teich, Fundamentals of Photonics (Wiley, New York, 2019), $3^{\mathrm{a}}$ ed.

[4] S.C. Zilio, Óptica Moderna Fundamentos e Aplicações (Instituto de Física de São Carlos, São Carlos, 2009).

[5] P.A. Bélanger, Opt. Lett. 16, 196 (1991).

[6] J.T. Verdeyen, Laser Electronics (Prentice Hall, Englewood Cliffs, 1995), $3^{\mathrm{a}}$ ed.

[7] A. Yariv, Optical Electronics (Saunders College $\mathrm{Pu}-$ blishing, New York, 1991), $4^{\mathrm{a}}$ ed.

[8] W. Koechner, Solid-State Laser Engineering (Springer, Berlin, 1995), $4^{\mathrm{a}}$ ed.

[9] G. Cerullo, S. De Silvestri, V. Magni e L. Pallaro, Opt. Lett. 19, 807 (1994).

[10] E.A. Barbosa e N.D. Vieira Jr., Opt. Comm. 188, 205 (2001).

[11] X. Huang e M. Wang, Opt. Comm. 158, 322 (1998).

[12] F.A. Callegari e A.A. Freschi, Revista Brasileira de Ensino de Física 37, 3303 (2015).

[13] E. Hecht, Optics (Addison-Wesley, Amsterdam, 1990), $2^{\mathrm{a}} \mathrm{ed}$.

[14] W.J. Smith, Modern Optical Engineering (McGraw-Hill, New York, 2000), $3^{\text {a }}$ ed. 